Proc. Indian Acad. Sci. (Chem. Sci.), Vol. 96, No. 6, April 1986, pp. 523-532.

(C) Printed in India.

\title{
On some aspects of metallisation with special reference to iron group transition metal oxides
}

\author{
P GANGULY \\ Solid State and Structural Chemistry Unit, Indian Institute of Science, Bangalore 560012, India \\ A bstract. The metal-insulator transition in $3 d$ transition metal oxides such as the ternary \\ perovskite oxides as well as the binary oxides both as a function of composition and \\ temperature are reviewed. The description of metallisation in terms of a change in sign of the \\ temperature coefficient of resistance as well as conductivity at $0 \mathrm{~K}$ are considered in terms of \\ modern theories based on localisation. It is pointed out that there may be grounds to believe \\ that in the high temperature limit a resistivity less than $\approx 10^{-1} \mathrm{ohm} \mathrm{cm}$ may be associated with \\ metallic properties despite a negative temperature coefficient of resistance. The correlation \\ between magnetic and electrical properties have also been examined.
}

Koywords. Metallisation; $3 d$ transition metal oxides; localisation.

\section{Introduction}

Several inorganic and organic compounds are known to exhibit metallic behaviour (Rao and Edwards 1986, for a brief review of such systems). The interest is to examine whether there are common features associated with metallisation in these systems. The classical requirement for metallic behaviour has been a positive temperature coefficient of resistance (TCR) as distinct from semiconductors which show a negative TCR. The composition controlled metal-insulator transition is then thought to be signalled by a change in the sign of TCR as a function of composition. However, in as much as semiconductor is considered to exhibit activated transport behaviour an insulator would be one which exhibits zero conductivity at $0 \mathrm{~K}\left(\sigma_{0}\right)$. A metal would then be one which shows a finite $\sigma_{0}$. In the latter case there may exist situations where the TCR is actually negative as discussed recently (Mott 1985, Mott and Kaveh 1985). By this definition the concept of metal-insulator (MI) transition has become blurred and there are several problems that are yet to be understood.

In this article we shall discuss the metallisation in oxides with special reference to the oxides of the iron group transition metals. The small radial extension of the $3 d$ electrons makes the bonding mainly ionic so that the bandwidths are expected to be narrow. The electrical transport and magnetic properties are considered to be due to electrons of predominantly $3 d$ character. A strong correlation between magnetic and electrical properties is therefore anticipated. We shall examine both the composition-controlled and temperature-induced $\mathrm{MI}$ transition from the viewpoint of recent experimental and theoretical work.

Contribution No. 353 from the Solid State and Structural Chemistry Unit. 


\section{Universal characteristics}

Mott $(1967,1972,1985 \mathrm{~b})$ had quite early proposed a theory for the existence of a minimum metallic conductivity, $\sigma_{\min }$, using the theory of localisation proposed by Anderson (1958). According to this theory, the high-temperature intercept of the $\log \sigma$ vs $1 / T$ plot of compositions which are insulating would correspond to the value of $\sigma_{\min }$ which is given by

$$
\sigma_{\min }=(1 / 3) g^{2} e^{2} / \hbar a \simeq 0.03 e^{2} / \hbar a
$$

where $g \sim(1 / 3)$ and $a$ is the separation between charge carriers. The change in sign of TCR occurs around the value of $\sigma<\sigma_{\min }$. The situation envisaged by Mott is shown in figure 1. The concept of $\sigma_{\min }$ is based on the assumption that the mean free path $l$ cannot be less than the interatomic spacing $a$. Without disorder this can be shown (Ioffe and Regel 1960$)$ to be nearly equal to $(1 / 3) e^{2} / \hbar a$. The factor $g$ in (1) is introduced to account for disorder, as a decrease in the density of states $\left(N_{E}\right)$ at $E_{F}$ is expected over that without disorder $\left(N_{E}^{\circ}\right)$, with $g=\left(N_{E}\right) /\left(N_{E}^{\circ}\right)$. In concentrated systems $a \sim 2-4 \mathrm{~A}$ and $\sigma_{\min } \sim 200-500 \mathrm{ohm}^{-1} \mathrm{~cm}^{-1}$. One of the early predictions of Mott was that $\sigma_{0}$ would drop discontinuously to zero through a composition-controlled MI transition when $\sigma$ becomes less than $\sigma_{\min }$. At low temperatures the insulating compositions are presumed to show in this model variable range hopping (Mott 1969) with $\sigma \propto \exp \left(-A / T^{1 / 4}\right)$ for three-dimensional systems.

Recently, however, scaling theories (Abrahams et al 1979, 1980; Ramakrishnan 1985; Lee and Ramakrishnan 1985) have shown that $\sigma_{\min }$ does not exist in the sense that there is no discontinuous decrease of $\sigma_{0}$ to zero. In this theory a dimensionless conductance $G(L)$ is defined for a cube of side $L\left[G(L)=\left(L h / e^{2}\right) \sigma(L)\right]$. A function $\beta(G)$ can then be defined such that $\beta(G)=\mathrm{d} \ln G(L) / \mathrm{d} \ln L$. This function is shown to be a universal function of $G$ only. In three dimensions $\beta$ has a zero at a value $G_{c}$. Then $G$ is a constant $G_{c}$ in the neighbourhood of zero and this should be an universal constant. Thus

$$
\sigma=\left(e^{2} / \hbar\right) G_{c} / L \text {. }
$$

The above equation leads to a vanishing conductivity as $L$ tends to infinity. This contradicts the predictions of Mott. In the Anderson (1958) model, Mott had argued that for a limiting value of $V / B$, (where $\pm \frac{1}{2} V$ are the limits of the random potential well

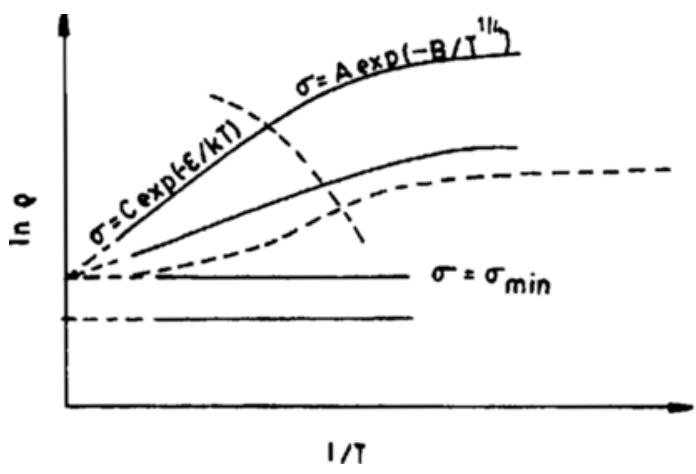

Figure 1. Expected changes in resistivity as a function of composition during a composition-controlled MI transition (From Mott and Kaveh, 1985). 
and $B$ is a bandwidth) all states are localised upto a limiting energy called the mobility edge. As $V / B$ increases the density of states at $E_{F}$ till at the limiting value it approaches the value $g$ in (1). Thus the conductivity at the mobility edge in Mott's model is given by (1). Equation 2, however, shows that at the mobility edge $g$ vanishes so that there can be no minimum metallic conductivity. Physically, this is due to an effect called incipient localisation' arising out of quantum interference effects (Bergmann 1983) of the two paths that an electron can have under time reversal symmetry. This backward scattering causes an electron diffusing out of a point to return to the original point thus adding to localisation effects.

Mott has proposed that at finite temperatures

$$
\sigma_{\min }=0.03 e^{2} / \hbar L_{i}
$$

where $L_{i}$ is the distance that an electron diffuses before suffering an inelastic collision. According to Mott and Kaveh (1985) eq. (3) is the limiting value of the conductivity as the localisation length, increases, since the conductivity would be determined by the value of $L_{i}$ rather than $\xi$ which is the distance in which the wave functions are little changed, $L_{i}$ is assumed to decrease with increasing temperature and approaches the value $a$ at high temperatures so that in the high temperature limit (1) is recovered. The exact temperature dependence of $L_{i}$ is not known. Mott and Kaveh (1985) have proposed that $L_{i}$ varies as $1 / T$ when electron-electron collisions are involved.

Most of Mott's arguments are based on the band picture. When $V / B$ is greater than the limiting value the localised states reside within the band tails. These localised charges carry current through phonon-assisted hopping. In an alternate model for charge conduction polarons are sometimes postulated (Emin 1983). In this model the atoms in the vicinity of a charge carrier are displaced to produce a potential well in which the charge carrier is self-trapped. When only a few atoms are involved these are called small polarons, the spatial extent of the charge carrier being small. The small polaron is favoured as the residence time of a charge carrier at a site increases. Small polaron bands have very narrow band widths. The formation and electronic transport properties of these polarons have been discussed recently by Emin (1983). The description of electrical transport in these systems is based on the Einstein relation $\sigma$ $=n e^{2} D / k T$ where $D$ is the diffusion constant $\left(=a^{2} v\right)$ with $v=v_{0} \exp \left(-E_{a} / k T\right)$. In the limit of the activation energy $E_{a}=0$ the maximum conductivity for small polaron hopping is expected to be around $10^{1} \mathrm{ohm}^{-1} \mathrm{~cm}^{-1}$ (Frohlich 1954; Bosman and van Daal 1970). It is to be noted that the ionic conductivity in superionic conductors has an upper limit close to the value of $1-10 \mathrm{ohm}^{-1} \mathrm{~cm}^{-1}$. This value of the conductivity is restricted by the attempt frequency, $v$, which is of the order of $10^{12} \mathrm{sec}^{-1}$, the phonon frequency. The attempt frequency, $v$, may be related to the exchange integral $J$ through $J=h v$ as proposed by Zener (1951) and Zener and Heikes (1953), Zener obtains an expression for the conductivity as

$$
\begin{aligned}
\sigma & \simeq\left(n e^{2} J a^{2} / h k T\right) \exp \left(-E_{a} / k T\right) \\
& \simeq e^{2} J / h a k T,
\end{aligned}
$$

when $E_{a}=0, v=J / h$ and $n=\left(1 / a^{3}\right)$. Equation 4 gave a close estimate of the value of the conductivity for several double exchange systerns. It is interesting to note that when $J$ is of the order of $k T$, (4) gives the Ioffe-Regel (IR) limit, $\sigma_{\mathrm{IR}}\left(\simeq e^{2} / 3 \hbar a\right)$. Zener's derivation would seem to imply that the attempt frequency in (4) can be related to the exchange frequency. 


\section{Composition-controlled metal-insulator transition}

The composition-controlled $\mathrm{MI}$ transition in oxides from the viewpoint of $\sigma_{\min }$ were first examined in $\mathrm{La}_{1-x} \mathrm{Sr}_{x} \mathrm{VO}_{3}$ and $\mathrm{Ln}_{n-x} \mathrm{Sr}_{x} \mathrm{VO}_{3}$ (Mott 1972; Rao et al 1977; Rao and Om Parkash 1977). In these systems disorder is created by a random distribution of $\mathrm{Sr}^{2+}$ and the disordering potential is the difference in the charge potential. The $\mathrm{ki}$ transition is considered to be due to delocalisation of $\mathrm{Co}^{4+}$ or $\mathrm{V}^{4+}$ holes which are bound to $\mathrm{Sr}^{2+}$ for small values of $x$. Predictions based on the Anderson model were substantiated by the observation of a linear $\log \rho$ vs $T^{-1 / 4}$ plot at low temperatures. The value of the conductivity at which TCR changes sign is around $500 \mathrm{ohm}^{-1} \mathrm{~cm}^{-1}$. These observations were consistent with the postulates of Mott. The intriguing feature is the nearly universal value of the critical composition $x_{c}(\simeq 0.28 \pm 0.03)$ at which TCR changes sign. In $\mathrm{La}_{1-x} \mathrm{~K}_{x} \mathrm{VO}_{3}$ (Bazov et al 1982) $x_{\mathrm{c}} \approx 0.13$ which seems to indicate that the critical parameter is the percentage of $\mathrm{B}^{4+}$ ions.

The $\mathrm{MI}$ transition in the $\mathrm{LaNi}_{1-x} B_{x} \mathrm{O}_{3}$ system $(B=\mathrm{Cr}, \mathrm{Mn}, \mathrm{Fe}, \mathrm{Co})$ have been studied recently (Ganguly et al 1984). $\mathrm{LaNiO}_{3}$ is metallic while the $\mathrm{LaBO}_{3}$ compounds are magnetic insulators (see Goodenough 1972). The $\mathrm{Ni}^{3+}$ and $B^{3+}$ ions have the same charge but are likely to have different $3 d^{n}$ energies. Any disorder is therefore likely to arise from changes in the exchange potential. In these systems also, it is observed that TCR changes sign around the value of $\sigma \sim 500 \mathrm{ohm}^{-1} \mathrm{~cm}^{-1}$. Localisation effects are most pronounced with $\mathrm{Mn}^{3+}$ and $\mathrm{Cr}^{3+}$. An examination of the energy level diagrams of Mizushima et al (1979) for the $3 d^{n}$ metal ions in $\mathrm{TiO}_{2}$ shows that $\mathrm{Mn}^{3+}$ and $\mathrm{Cr}^{3+}$ ions are furthest energetically from that of $\mathrm{Ni}^{3+}$. This could account for their strong localising influence (see Rao and Ganguly 1985a, 1985b). Several other systems such as $\mathrm{LaRu}_{1-x} B_{x} \mathrm{O}_{3} \quad(B=\mathrm{Ga}, \mathrm{Fe})$ show changes in the sign of TCR around $\sigma$ $\sim 300-500 \mathrm{ohm}^{-1} \mathrm{~cm}^{-1}$ (Bouchard et al 1973, 1977).

The compositions with $x$ close to $x_{\mathfrak{c}}$ and with negative TCR could nevertheless be metallic in the sense of a finite $\sigma(0)$. There are several indicators to this behaviour:

(i) We have observed (Ganguly et al 1984) that the conductivity in the $\mathrm{LaNi}_{1-x} B_{\mathrm{x}} \mathrm{O}_{3}$ systems in the range $15-300 \mathrm{~K}$ could be fitted to an empirical expression of the type

$$
\sigma=A \exp \left[-E_{a} / k(T+\theta)\right],
$$

where $\theta$ is positive and $A$ approaches $\sigma_{\min }$ as $x$ approaches $x_{c}$. A finite value of $\theta$ implies a finite $\sigma_{0}$. The above fit has been subsequently found to be successful in several other oxide systems. Extension of resistivity studies down to lower temperatures is under way to examine the existence of $\sigma_{0}$ as well the validity of (5).

(ii) An infra-red study in the range $1000-300 \mathrm{~cm}^{-1}$ of $\mathrm{LaNi}_{1-x} B_{x} \mathrm{O}_{3}$ showed (Ganguly and Vasanthacharya 1986) that features due to localised optic modes disappear when the resistivity of the system decreases below $\sim 10^{-1} \mathrm{ohm} \mathrm{cm}$. This is about two orders of magnitude more than the value corresponding to $\sigma_{\min }(1)$ or the value at which TCR changes sign. Such behaviour has been observed in several other perovskite and layered perovskite oxide systems with the $\mathrm{K}_{2} \mathrm{NiF}_{4}$ structure.

(iii) In the $\mathrm{LaNi}_{1-x} \mathrm{Mn}_{x} \mathrm{O}_{3}$ system the $x=0.05$ and 0.10 compositions show nearly temperature-independent resistivity below $40 \mathrm{~K}$ although at higher temperatures they show a negative rCR. This would imply a finite $\sigma_{0}$. These systems show anomalous magnetic susceptibility behaviour below $40 \mathrm{~K}$ (Vasanthacharya et al 1984a). This behaviour has been associated with itinerant electron behaviour as the more insulating $\mathrm{LaMn}_{x} \mathrm{Co}_{1-x} \mathrm{O}_{3}$ system does not show such behaviour although Co-O-Mn interac- 
tions are ferromagnetic just as the Mn-O-Ni interactions. We note that the $x \leqslant 0.01$ compositions in the $\mathrm{LaNi}_{1}-{ }_{x} \mathrm{Mn}_{x} \mathrm{O}_{3}$ systems show a resistivity at low temperatures $\sim 10^{-1} \mathrm{ohm} \mathrm{cm}$ while in the corresponding $\mathrm{LaCo}_{1-x} \mathrm{Mn}_{x} \mathrm{O}_{3}$ system $P>10^{4} \mathrm{ohm} \mathrm{cm}$.

\section{Temperature induced metal-insulator transition}

For pure compounds where there is no disorder the concept of localisation or $\sigma_{\min }$ is not really applicable. Many of the $3 d$ transition metal oxides such as $\mathrm{CrO}_{2}, \mathrm{TiO}, \mathrm{SrCrO}_{3}$, $\mathrm{SrFeO}_{3}, \mathrm{LaNiO}_{3}$ etc., which are metallic, have resistivities in the range $10^{-3}-10^{-4} \mathrm{ohm} \mathrm{cm}$ at ordinary temperatures (see Rao and Rao 1970 and references therein). However, compounds such as $\mathrm{LaCoO}_{3}, \mathrm{LaTiO}_{3}, \mathrm{La}_{2} \mathrm{NiO}_{4+\delta}, \mathrm{La}_{2} \mathrm{CuO}_{4}$ etc., (Goodenough 1972, Rao and Rao 1970, Rao and Ganguly 1985a, 1985b, and references therein) which are considered to be metallic at high temperatures with positive TCR, are semiconducting with a negative TCR at lower temperatures. In these compounds, the value of the resistivity at higher temperatures is greater than that $(\sim 2000 \mu \mathrm{ohm} \mathrm{cm})$ corresponding to $\sigma_{\min }$ in disordered systems. Like the Mooij criterion applicable to amorphous metals (Mooij 1973) a criterion for the oxides as well as for other compounds (Rao and Ganguly 1986) may be put forward, i.e., there is a limiting value of the resistivity $(\sim 2000 \mu \mathrm{ohm} \mathrm{cm})$ for compounds which delineates materials which exhibit a positive TCR from those which exhibit a negative TCR in the absence of a structural distortion. In the case of amorphous metals the limiting value is $\sim 200 \mu \mathrm{ohm} \mathrm{cm}$.

The question arises as to what the values of the conductivity at which metallisation is observed are on treating temperature as a disordering field. Some of the most dramatic changes in the resistivity in the temperature-induced MI transitions are shown by $3 d$ transition metal oxides especially the oxides of vanadium and titanium such as $\mathrm{VO}_{2}$, $\mathrm{V}_{n} \mathrm{O}_{2 n-1}, \mathrm{Ti}_{n} \mathrm{O}_{2 n-1}$. In these oxides the metallic phase has a conductivity of the order of $10^{3} \mathrm{ohm}^{-1} \mathrm{~cm}^{-1}$ which is just larger than the value of $\sigma_{\min }$ expected for these compounds from (1). On the other hand, in $\mathrm{Fe}_{3} \mathrm{O}_{4}$ the resistivity after the Verwey transition is of the order of $10^{-2} \mathrm{ohm} \mathrm{cm}$ which is less than the value of $\sigma_{\min }$ expected. In this compound, the resistivity shows a negative TCR after the Verwey transition until the magnetic ordering temperature above which it is nearly temperature-independent (Miles et al 1957). The conductivity at this temperature is around $200 \mathrm{ohm}^{-1} \mathrm{~cm}^{-1}$ (see Aragon et al 1985 and references therein). Thus the high temperature metallisation of materials seems to show that there could be a limiting value of the conductivity for metallisation.

The very careful experimental work on single crystals of $\mathrm{V}_{2-x} \mathrm{Cr}_{x} \mathrm{O}_{3}$ and $\mathrm{Fe}_{3} \mathrm{O}_{4-\delta}$ (Kuwamoto et al 1980; Aragon et al 1985 and references therein). In the $\mathrm{V}_{2-x} \mathrm{Cr}_{x} \mathrm{O}_{3}$ system it is known that the $x=0$ composition shows a sharp Mi transition at $150 \mathrm{~K}$ the conductivity in the metallic phase being around $1000 \mathrm{ohm}^{-1} \mathrm{~cm}^{-1}$. As $x$ is increased the MI transition temperature $\left(T_{\mathrm{MI}}\right)$ increases. The resistivity in the metallic regime is also increased. At still higher values of $x$ there is a temperature $T_{\mathrm{IM}}\left(>T_{\mathrm{MI}}\right)$ at which there is a sharp increase in resistivity by several orders of magnitude when $T>T_{\mathrm{IM}}$ the material is semiconducting. This temperature $T_{\mathrm{IM}}$ is decreased as $x$ is further increased so that the range of metallic behaviour $T_{\mathrm{IM}}>T>T_{\mathrm{MI}}$ decreases with increase in $x$ and disappears above a particular value $x_{r}$. These transitions have been treated in an elegant manner recently by Honig and coworkers (see Honig and Spalek 1986) employing 
essentially a Brinkmann-Rice (1970) model and taking into account the free energy contributions from the spin. This study makes a serious and significant contribution to the understanding of temperature induced MI transitions. What is of interest to us is that the re-entrant metallic behaviour is most marked when the resistivity in the metallic phase is close to $\sigma_{\min }$ of (1) and that the metallic phase is not seen when the resistivity in the region $T_{\mathrm{MI}}<T<T_{\mathrm{IM}}$ becomes greater than $\sim 10^{-1} \mathrm{ohm} \mathrm{cm}$. Thus the results of the $\mathrm{V}_{2-x} \mathrm{Cr}_{x} \mathrm{O}_{3}$ system seem to show that metallicity at high temperatures may be defined when $\rho$ is less than or equal to $10^{-1} \mathrm{ohm} \mathrm{cm}$. In the $\mathrm{Fe}_{3} \mathrm{O}_{4-\delta}$ system the Verwey transition is first order with a finite enthalpy of transition (see Honig 1986), for small values of $\delta$. The magnitude of the resistivity change at the first order transition (corresponding to the Verwey transition) decreases as $\delta$ is increased. The resistivity after the transition is less than $10^{-1} \mathrm{ohm} \mathrm{cm}$. At higher values of $\delta$ the transition becomes second order. Interestingly, this happens as the value of the resistivity in the high temperature phase becomes more than $\sim 10^{-1} \mathrm{ohm} \mathrm{cm}$. Thus a value of $10^{-1} \mathrm{ohm} \mathrm{cm}$ seems to be important at least at high temperatures. In the $\mathrm{LaNi}_{1}-\mathrm{B}_{x} \mathrm{O}_{3}$ and $\mathrm{La}_{1-x} \mathrm{Sr}_{x} \mathrm{BO}_{3}$ systems referred to earlier there are also indications that at high temperatures the resistivity behaviour is not as predicted by Mott (Figure 1) but instead tends to become temperature independent before it reaches the value of the resistivity corresponding to the high-temperature intercept. This is seen for example in the high temperature resistivity studies on $\mathrm{LaNi}_{1-{ }_{x}} \mathrm{Fe}_{x} \mathrm{O}_{3}$ in figure 2 (Vasanthacharya 1985) and for the $\mathrm{La}_{1-x} \mathrm{Sr}_{x} \mathrm{CoO}_{3}$ samples (Rao and Om Parkash 1977). The resistivity behaviour in figure 2 is closer to that predicted from the percolation edge model.

In two-dimensional oxides with $\mathrm{K}_{2} \mathrm{NiF}_{4}$ structure it has been observed that in several systems such as $\mathrm{LaSrNi}_{1-x} \mathrm{Fe}_{x} \mathrm{O}_{4}, \mathrm{La}_{1-x} \mathrm{Sr}_{1+x} \mathrm{CoO}_{4}, \mathrm{LaSrNi}_{1-x} \mathrm{Al}_{x} \mathrm{O}_{4}$ (Singh 1982; Ganguly 1986) the high temperature intercepts of the $\log \rho$ vs $1 / T$ plots extrapolate to a

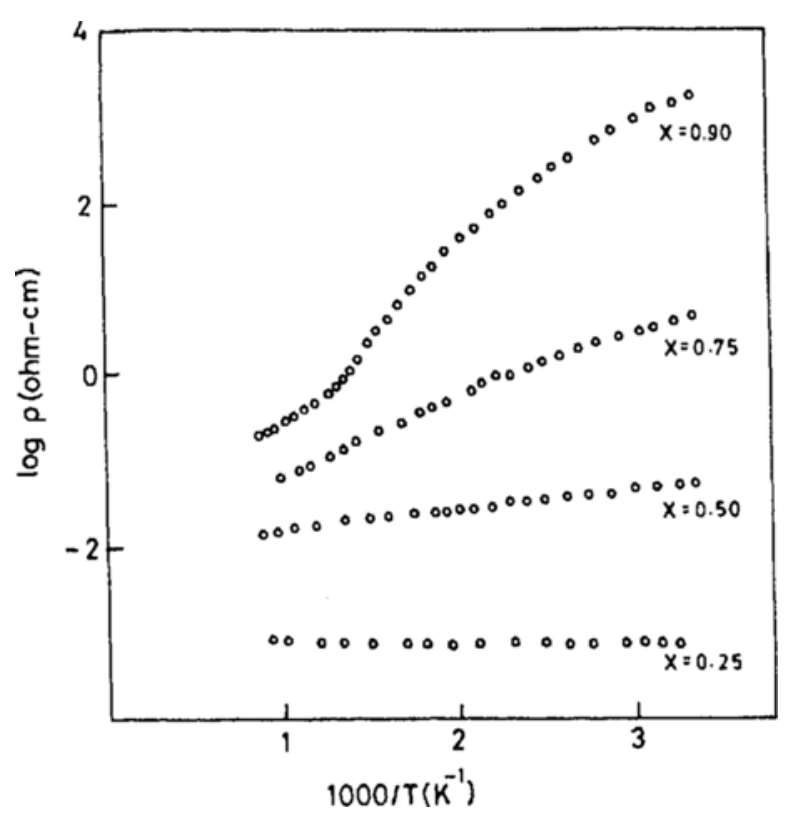

Figure 2. $\log \rho$ vs $1 / T$ plots of $\mathrm{LaNi}_{1-x} \mathrm{Fe}_{x} \mathrm{O}_{3}$ (from Vasanthacharya 1985). 
value of $\sigma \sim 10^{2} \mathrm{ohm}^{-1} \mathrm{~cm}^{-1}$ so that the high temperature $\sigma_{\min }$ seems to be reduced over that of the corresponding three-dimensional perovskites. In the $(\mathrm{LaO})_{n+1}$ $\left(\mathrm{LaNiO}_{3}\right)_{n}$ systems it was observed that $n=2$ and $n=3$ compositions had a nearly temperature-independent resistivity of $\sim 5 \times 10^{-2} \mathrm{ohm} \mathrm{cm}$ and $\sim 10^{-2} \mathrm{ohm} \mathrm{cm}$, respectively. These results are not consistent with the value of $\sigma_{\min }$ of (1) but it does suggest that metallicity at high temperatures may be associated with resistivities less than $10^{-1} \mathrm{ohm} \mathrm{cm}$.

\section{Changes in magnetic properties across metal-insulator transitions}

Systems which show a sharp mi transition at elevated temperatures such as $\mathrm{V}_{2} \mathrm{O}_{3}$ (see Honig 1985) show marked changes in the magnetic susceptibility at the transition. In the composition-controlled metal-insulator transitions the correlation between magnetic and electrical properties are not obvious. In the $\mathrm{La}_{1-x} \mathrm{Sr}_{x} \mathrm{VO}_{3}$ system compositions with negative TCR show evidence for antiferromagnetic ordering in terms of a maximum in the susceptibility (Dougier and Hagenmuller 1975). The compositions with positive TCR show nearly temperature-independent susceptibility at high temperatures. At lower temperatures there is an enhancement in the susceptibility. The behaviour is similar to that of $\mathrm{LaNiO}_{3}$ (Vasanthacharya et al 1984). The magnitude of the temperature-independent susceptibility is very large $\left(\sim 700 \times 10^{-6} \mathrm{emu} / \mathrm{mole}\right)$. If attributed to Pauli paramagnetism such a high value would indicate Fermi temperatures around $500-1000 \mathrm{~K}$ only or band widths of the order of only $0.1 \mathrm{eV}$. In $\mathrm{LaRuO}_{3}$ there is a strong temperature-dependence of the susceptibility (Bouchard et al 1973). Indeed in $\mathrm{LaRu}_{1-x} \mathrm{Ga}_{x} \mathrm{O}_{3}$ there is hardly any change in the nature of the high temperature susceptibility especially in the slopes of the $\chi^{-1}$ vs $T$ plots for all values of $x$. Similar behaviour has been found in the $\mathrm{LaNi}_{1}{ }_{x} \mathrm{Al}_{x} \mathrm{O}_{3}$ system for small values of $x$. In $\mathrm{LaNi}_{1-x} B_{x} \mathrm{O}_{3}$, samples with $x$ close to $x_{c}$ seem to show evidence for temperatureindependence of susceptibility (Vasanthacharya et al 1984). These samples have a resistivity less than $10^{-1} \mathrm{ohm} \mathrm{cm}$ at the lowest temperatures. Interestingly, we note that the $\rho$ vs $\log T$ plots of some of these samples are nearly linear (figure 3).

It does seem, therefore, that magnetic susceptibility is not clearly correlated with electrical transport properties. Indeed in systems such as $\mathrm{LaSrNiO}_{4}$ the magnetic susceptibility behaviour is very similar to that of $\mathrm{LaNiO}_{3}$ at high temperatures. Such similarities in the magnetic susceptibility behaviour in two-dimensional and threedimensional perovskites have been noticed (Singh 1982; Ganguly 1986). However, despite similar magnetic behaviour the resistivities of the two-dimensional systems are orders of magnitude higher than the corresponding metallic three-dimensional perovskites (Ganguly and Rao 1984; Ganguly 1986). Such behaviour is not anticipated if the magnetic $d$ electrons are also considered to be charge carriers since in the tightbinding approximation the band width is related to the transfer integral which also determines the magnetic properties.

\section{General remarks}

In several diverse systems such as doped polyacetylene, amorphous $\mathrm{Ge}-\mathrm{Fe}$ alloys, $\mathrm{Na}_{x} \mathrm{WO}_{3}$ bronzes etc. the temperature coefficient of resistivity changes sign around the 


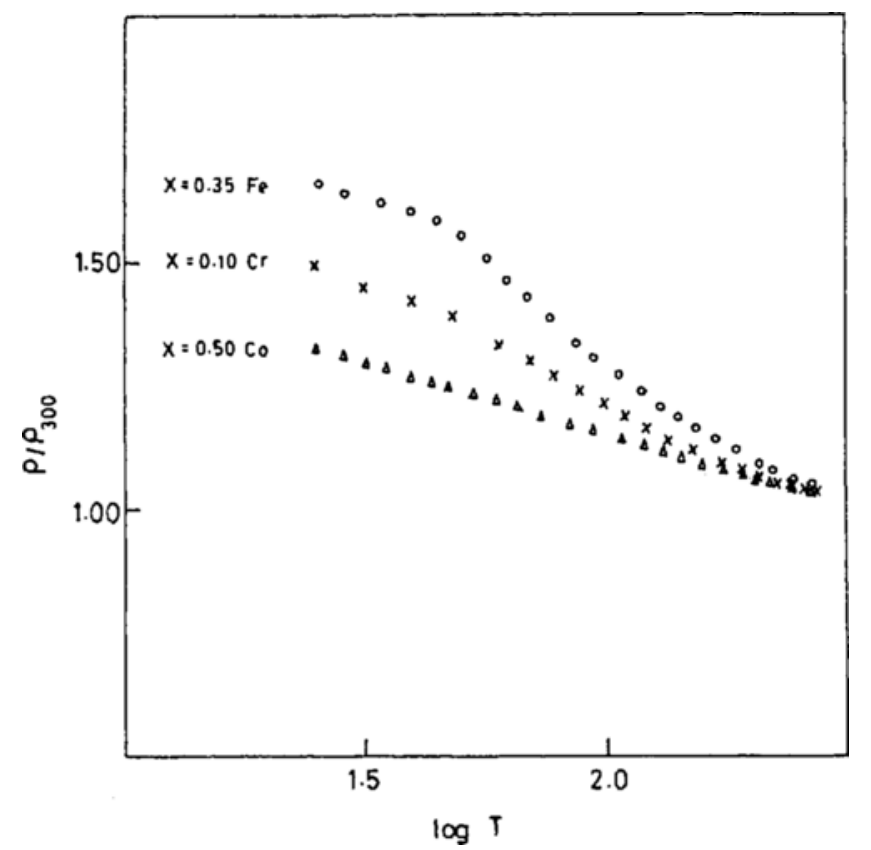

Figure 3. $\rho$ vs $\log T$ plots of some $\mathrm{LaNi}_{1-x} \mathrm{~B}_{x} \mathrm{O}_{3}$ for values of $x$ close to $x_{c}$ where TCR changes sign (from $N Y$ Vasanthacharya, unpublished results).

value predicted by (1). The value associated with $\sigma_{\min }$ is thus insensitive to the details of the crystal-field, spin magnitude, crystallinity etc. and thus seems to indicate a universal criterion. Equation 3 has the property of satisfying some of these results both in the high temperature and low temperature limits. At the same time materials with resistivities between $\sim 10^{-1}$ and $\sim 10^{-3} \mathrm{ohm} \mathrm{cm}$ would seem to be associated with metallic rather than localised electron behaviour despite a negative TCR. The negative TCR is due to the change in $L_{i}$. Mott and Kaveh (1985) have proposed that for electron scattering $L_{i}$ varies as $1 / T$. The results in figure 3 would seem to suggest that it is $\mathrm{d} L_{t} / \mathrm{d} T$ which varies as $1 / T$. On the other hand, since a linear $\rho$ vs $\log T$ plot is reminiscent of the Kondo effect one could argue that a Kondo type of bound state formation leads to an increase in resistivity with decreasing temperature. However, the Kondo mechanism is not properly understood for such poorly conducting systems.

In a qualitative sense the limits of $\sim 10^{-1} \mathrm{ohm} \mathrm{cm}$ as the minimum activated resistivity and $\sim 10^{-3} \mathrm{ohm} \mathrm{cm}$ as the maximum metallic conductivity (with positive TCR) may be understood from residence time arguments. It was shown earlier (Rao and Ganguly $1985 \mathrm{a}$, b) that by proposing a minimum relaxation time $\tau_{\min } \sim 10^{-14} \mathrm{sec}$ we may obtain a value of $\sigma_{\min }$ from the Boltzmann formula

$$
\sigma=n e^{2} \tau / m^{*},
$$

close to that predicted from (1) assuming $m^{*}=10 m_{0} \cdot n / m^{*}$ may therefore be taken as the fraction of charge carriers. $1 / f$ noise studies (Prasad et al 1979) on $\mathrm{La}_{1-x} \mathrm{Sr}_{x} \mathrm{VO}_{3}$ have shown that in the metallic state the fraction of charge carriers is around $10 \%$. From Mott's criterion $n_{c}^{1 / 3} a_{H} \sim 0-25$ for metallisation $\left(n_{c}\right.$ is the critical charge carrier 
concentration per unit volume and $a_{H}$ is the Bohr radius) the fraction of the volume occupied by the charge carriers with a radius $a_{H}$ is $\sim 10 \%$ of the total volume available from close-packing considerations. In concentrated systems therefore only ten $\%$ of the charge carriers need be ionised for Mott's criterion to be satisfied. Interestingly, if we define an effective radius $a_{\text {eff }}$ such that (4/3) $\pi n_{c} a_{\text {eff }}^{3}=0.74$ (the close-packed volume) then $a_{\mathrm{eff}} / a_{H}=2.15$ which is close to the ratio of the van der Waals radius of hydrogen atom and its Bohr radius.

An effective mass $m^{*} \approx 10 m_{0}$ corresponds to $a$ band width of $0.1 \mathrm{eV}$ which is the value that we obtained from the temperature-independent part of the susceptibility of $\mathrm{LaNiO}_{3}$ and $\mathrm{La}_{1-x} \mathrm{Sr}_{x} \mathrm{VO}_{3}$. From the uncertainty principle, such a band width corresponds to a residence time of $\sim 10^{-14} \mathrm{sec}$. Since this residence time is much less than a typical vibrational period of $10^{-12}$ to $10^{-13} \mathrm{sec}$ a $\tau_{\text {res }} \sim 10^{-14} \mathrm{sec}$ could define a free electron. This would justify the use of a $\tau_{\min } \sim 10^{-14} \sec$ in (6). With this definition of $\tau_{\min }$ for metallicity a metallic conductivity of $\sim 10 \mathrm{ohm}^{-1} \mathrm{~cm}^{-1}$ would correspond to an effective mass $m^{*} \sim 1000 m_{0}$ and a corresponding $\tau_{\text {res }} \sim 10^{-12}$ sec which is just the limit expected for metallisation. NMR experiments (see Warren 1985) on metallisation in liquids support the localisation time scales discussed above.

That a minimum bandwidth correspending to a minimum $\tau_{\text {res }}$ may be responsible for metallisation is also seen from the recent observation (Rao and Ganguly 1986) that for liquid elements a heat of vapourisation, $\Delta H_{v}$, of $\sim 0.4 \mathrm{eV}$ per mole distinguishes metallic liquids from the non-metallic ones. This simple criterion cannot of course be applied to all liquids. This also breaks down for elements such as halogens or chalcogens if the dissociation energy, $D$, is added to $\Delta H_{v}$ of the dimeric molecules in order to obtain the $\Delta H_{v}$ of the element as distinct from the molecule. Apparently, the critical factor seems to be the critical ratio of $\Delta H_{\mathrm{v}} / D(\sim 1 \cdot 6)$ for metallisation to occur.

\section{Acknowledgements}

The author would like to thank Professor C N R Rao for several discussions on this subject and for encouragement. He also would like to thank Drs S Ramasesha and B Bagchi for some illuminating discussions.

\section{References}

Abrahams E, Anderson P W, Licciardello D C and Ramakrishnan T V 1979 Phys. Rev. Lett. 42673 Abrahams E, Anderson P W and Ramakrishnan T V 1980 Philos. Mag. B42 827

Anderson P W 1958 Phys. Rev. 1091492

Aragon R, Shepherd J P, Konitzer J W, Buttrey D J, Rasmussen R J and Honig J M 1985 J. Appl. Phys. 57 3221

Bazov G V, Makarowa $O$ V and Shvekin P 1982 Inorg. Mater. 18720

Bergmann G 1983 Phys. Rev. B28 2914

Bosman A J and van Daal A J 1970 Adv. Phys. 191

Bouchard R J, Weiher J F and Gilsen J L 19'73 J. Solid State Chem. 6519

Bouchard R J, Weiher J F and Gilsen J L 1977 J. Solid State Chem. 21135

Brinkman W F and Rice T M 1970 Phys. Rev. 824302

Dougier P and Hagenmuller P $1975 \mathrm{~J}$. Solid State Chem. 15158

Emin D 1983 Comments Solid State Phys. 11 35, 59

Frohlich H 1954 Adv. Phys. 3375 


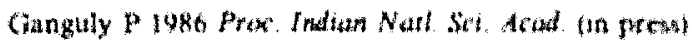

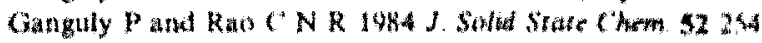

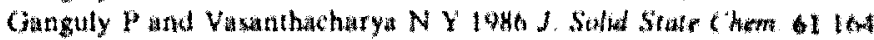

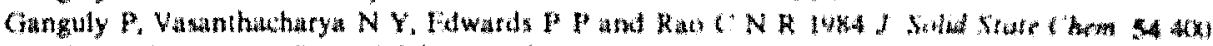
Goodenough J B 1972 Proy sibld Srote them 5145

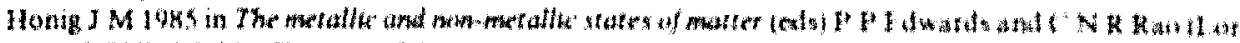

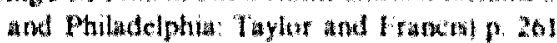

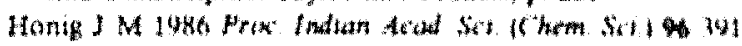

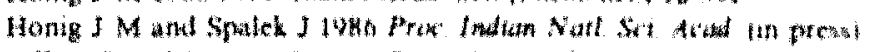

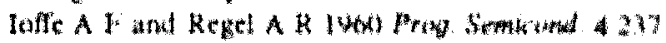

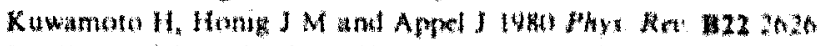

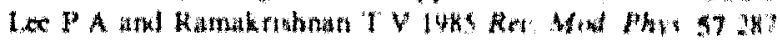

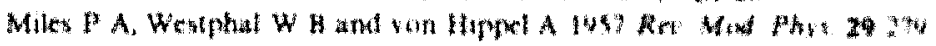

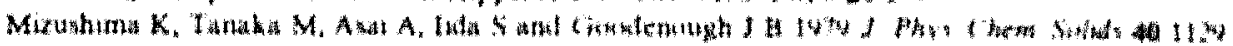

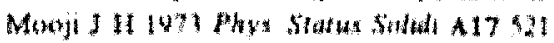

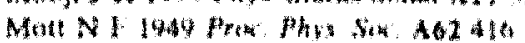

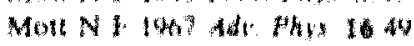

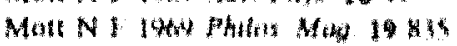

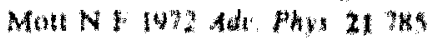

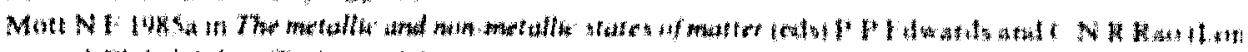

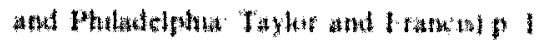

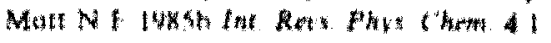

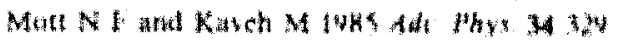

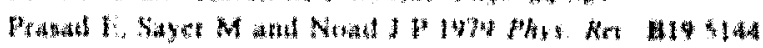

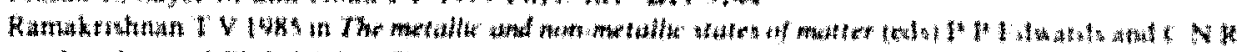

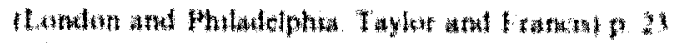

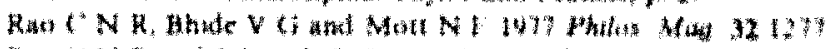

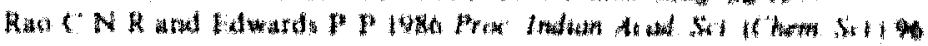

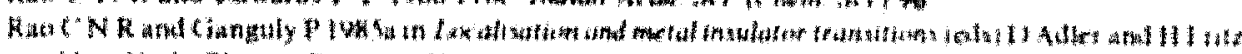

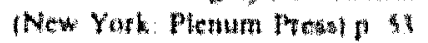

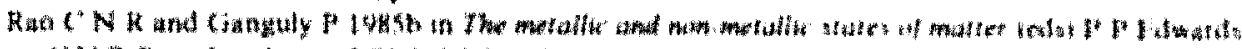

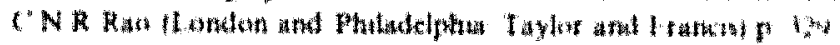

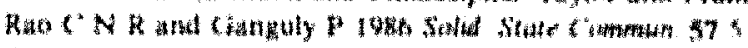

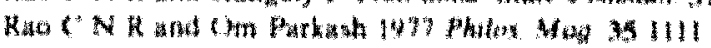

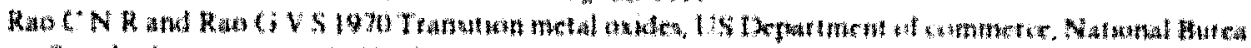

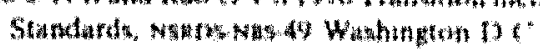

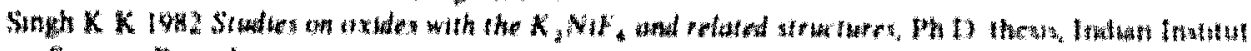
Sexence, Hungutare

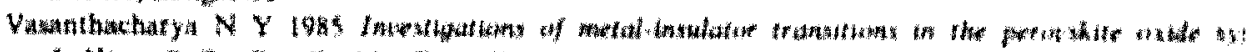

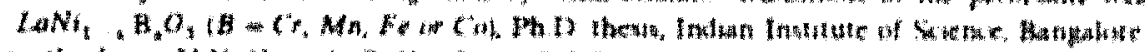

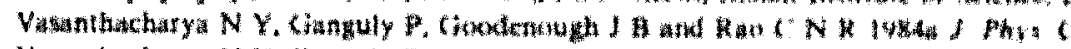

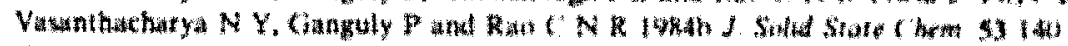

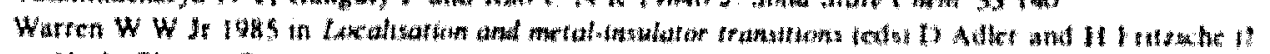

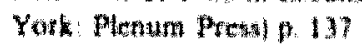

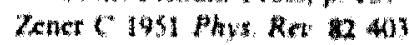

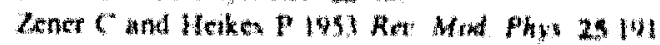

